\title{
Pain in cognitively impaired, non-communicating children
}

P Stallard, L Williams, S Lenton, R Velleman

\begin{abstract}
Aim-To detail the everyday occurrence of pain in non-communicating children with cognitive impairment.

Methods-Thirty four parents of cognitively impaired verbally non-communicating children completed pain diaries over a two week period. Each day, for five defined periods, parents rated whether their child had been in pain, and if so, its severity and duration.

Results-Twenty five $\mathbf{7 3 . 5 \% )}$ children experienced pain on at least one day, with moderate or severe levels of pain being experienced by $23(67.6 \%)$. Four children (11.7\%) experienced moderate or severe pain lasting longer than $\mathbf{3 0}$ minutes on five or more days. No child was receiving active pain management.

Conclusions-Everyday pain in children with severe cognitive impairment is common, yet is rarely actively treated. (Arch Dis Child 2001;85:460-462)
\end{abstract}

Keywords: pain; cognitive impairment; non-communicating

There is growing awareness of the psychological effects of unrelieved pain in children and the need to provide effective pain relief. ${ }^{12}$ Pain is common, with a community survey of children aged $0-18$ in the Netherlands identifying $54 \%$ who had experienced pain within the past three months. ${ }^{3}$ Chronic pain, defined as recurrent or continuous pain for more than three months, was found in $25 \%$, of whom $57 \%$ had consulted a physician and $39 \%$ had treated the pain with medication. ${ }^{4}$

Assessing pain in children is complex, particularly when the child is cognitively impaired and unable to communicate verbally. Neurological impairments may alter the neurological system and affect the child's ability to comprehend and communicate pain. ${ }^{5}$ Parents of children with cognitive impairment may feel that their children experience pain differently from those without cognitive impairment. ${ }^{6}$ Although parents may perceive their child as having a higher pain tolerance and a lower sensitivity, there is no conclusive evidence to suggest that children with cognitive impairments are insensitive or indifferent to pain. ${ }^{5}$ Children with severe cognitive impairments have a higher risk of experiencing pain, while their communication difficulty increases the likelihood that it will go unrecognised and so remain untreated. ${ }^{7}$ A survey of parents of 93 children with non-malignant life threatening conditions found $60 \%$ assessed their child to experience severe pain or discomfort that was not being effectively managed. ${ }^{8}$ Over half of these children were severely cognitively impaired and unable to communicate verbally.

The assessment of pain in noncommunicating children relies extensively on third party observations. Parents can make an important contribution to this process, although their knowledge is often overlooked. ${ }^{9}$ They know their child well, are sensitive to subtle changes in behaviour that may indicate pain, and with communicating children, reasonable correlations between parent and child reports of pain have been reported. ${ }^{10}$ Parents of non-communicating children with severe cognitive impairment are confident that they can detect pain, ${ }^{11}$ inferring it from indirect cues such as crying, facial expression, body movements, or behavioural and emotional changes. ${ }^{6}$ Consistent findings are emerging from research groups, suggesting that this population use a core set of cues to express pain. ${ }^{11-13}$

To date, research in children's pain has tended to focus on the assessment of acute pain, typically in hospital settings, over a very defined time period in response to a specific painful event. The frequency and severity of everyday pain experienced by more vulnerable groups of children in community settings has not been detailed. Our purpose is to detail the everyday occurrence of pain in noncommunicating cognitively impaired children with a chronic life threatening illness.

\section{Participants and methods}

Ethical consent for the study was obtained from the local ethical committee. Eligible children were identified from the database of the Lifetime Service, a community nursing and psychological service in Bath, UK for children with a non-malignant, life threatening illness. Children were included in the study if they had no expressive language (or alternative communication language such as Makaton) and were aged 2 or over. The carers of 41 children were invited to participate, of whom 34 (82.9\%) agreed. The children were 18 boys, 16 girls 
with an average age of 9.4 years. Eighteen had a primary diagnosis of a static central nervous system disorder (for example, cerebral palsy, spina bifida, post-encephalitis), seven progressive central nervous system disorder (for example, mucopolysaccharidosis, Rett syndrome), and nine chromosomal or other disorders (for example, partial trisomy of chromosome 13, Goldenhar syndrome).

The study was prospective and over a two week period the carer assessed the presence, severity, and duration of pain in their child. Each day the carer was asked to record whether their "child was in pain at any time" during the five specified rating periods of 800 am until $1200 \mathrm{pm}, 1200 \mathrm{pm}$ to $400 \mathrm{pm}, 400 \mathrm{pm}$ until $800 \mathrm{pm}, 800 \mathrm{pm}$ until $1200 \mathrm{am}$, and 1200 am until $800 \mathrm{am}$. If the child was in pain, the carer was asked to rate on a three point Likert scale its severity (mild, moderate, or severe) and duration during the rating period (up to 10 minutes, 10-30 minutes, over 30 minutes).

\section{Results}

During the two week observation period, nine $(26.5 \%)$ children were assessed as experiencing no pain, and $25(73.5 \%)$ as experiencing pain on at least one day. Of those considered to be in pain, $21(84 \%)$ were judged to experience pain on five or more separate days, with eight $(32 \%)$ experiencing pain on 12 or more days. Most of those who experienced pain $(22,88 \%)$ suffered on more than one consecutive day. On average, children experienced pain on 6.05 consecutive days (range 2-14 days), with 13 $(52 \%)$ being in pain on four or more consecutive days. Although most experienced pain on at least one day during the observation period, the pain was typically confined to one of the five daily rating periods $(x=1.18)$. Table 1 summarises the time, duration, and severity of the 415 recorded pain episodes.

Of the 74 pain episodes that lasted longer than 30 minutes, $25(33.8 \%)$ occurred during the period from $1200 \mathrm{am}$ until $800 \mathrm{am}$. This was also the most frequent time for moderate or severe pain, with $56(26.9 \%)$ of the recorded episodes occurring during this period.

Moderate or severe pain episodes were experienced by $23(67 \%)$ children. Of these, 13 $(56.5 \%)$ experienced moderate or severe pain on five or more separate days, with four

Table 1 Time, severity, and duration of pain episodes $(n=415)$

\begin{tabular}{lr}
\hline & Number (\%) \\
\hline Time of pain & $98(23.6)$ \\
$800 \mathrm{am}$ to $1200 \mathrm{pm}$ & $84(20.2)$ \\
$1200 \mathrm{pm}$ to $400 \mathrm{pm}$ & $60(14.5)$ \\
$400 \mathrm{pm}$ to $800 \mathrm{pm}$ & $86(20.7)$ \\
$800 \mathrm{pm}$ to $1200 \mathrm{am}$ & $87(21.0)$ \\
1200 am to $800 \mathrm{am}$ & \\
Severity of pain & $207(49.9)$ \\
Mild & $160(38.6)$ \\
Moderate & $48(11.5)$ \\
Severe & \\
& \\
Duration of pain & $183(44.1)$ \\
Up to 10 minutes & $158(38.1)$ \\
10-30 minutes & $74(17.8)$ \\
More than 30 minutes & \\
\hline
\end{tabular}

(17.4\%) suffering pain at these levels for 11 or more days. Most pain episodes (55.9\%) were judged to last longer than 10 minutes, with $12 / 25(48 \%)$ children having episodes lasting longer than 10 minutes on five or more days.

Pain appeared to be a particular problem for four $(11.7 \%)$ children, who had moderate or severe pain lasting longer than 30 minutes on five or more days during the two week observation period.

Finally, although the experience of pain was common among this group of children, none were receiving active treatment for its relief or management.

\section{Discussion}

Our preliminary findings suggest that pain in non-communicating children is common. Comparative data with community samples is unfortunately lacking, although the results from the Netherlands study suggest that approximately half of all children will experience pain at some time over a three month period. ${ }^{4}$ In our study, two thirds of cognitively impaired non-communicating children were assessed by their carers to experience moderate or severe pain on at least one day over a considerably shorter two week period.

The length of time these children had experienced pain was not assessed, so we cannot assess the proportion of children experiencing chronic and persistent pain. Previous research we have undertaken with this client group would lead us to suspect that many have chronic pain, which parents often identified as starting during the first year of their child's life. ${ }^{13}$ We are therefore surprised that no child we studied was receiving any specific pain relief. This is in stark contrast to research with community samples, where almost $40 \%$ of those with chronic pain had received medication. ${ }^{4}$ We do not know whether this difference was because parents had not sought specific help for their child's pain or because of professional beliefs and attitudes about pain in cognitively impaired children. Parents are undoubtedly concerned about pain and distress in their children, although it is possible that consultations with medical staff may be dominated by other more pressing issues. In terms of beliefs, professional perception of pain and use of medication as assessed by pain vignettes was not found to be affected by cognitive impairment in the child. ${ }^{14}$ Whether these beliefs are an accurate reflection of clinical practice has yet to be determined. Although the reasons are unclear, our findings would suggest that while pain in cognitively impaired children is more common than within the normal population, verbally non-communicating children are less likely to receive active pain management.

In considering these findings, the limitations of our study need to be acknowledged. Firstly, the sample size was small, so a larger, more robust study is needed to substantiate these findings. Secondly, it is possible that our participants may have been more sensitive to childhood pain, which may have contributed to the raised rates found in our study group. 
While this is possible, the Netherlands community survey adopted a similar methodology for children under the age of 7 and relied on parental report. ${ }^{3}$ Furthermore, research would suggest that parents underestimate, rather than overestimate, levels of pain in their children. ${ }^{15}$ Regardless of whether these results under- or overestimate pain in non-communicating children, it is clear that pain is an important clinical issue for this group.

Failure to identify and treat pain causes unnecessary distress and suffering for the child, and increases parental and family stress. Parents are often frustrated or frightened that their child's pain is not diagnosed or treated. ${ }^{16}$ Persistent childhood pain is associated with poor physical health and emotional disorder in the parents. ${ }^{17}$ The potential effects on everyday life are substantial, and we note that the longest and more severe episodes of pain in this study occurred between midnight and 800 am, which will obviously disrupt sleep. Pain may therefore be an important contributing factor to the raised rates of poor parental mental health and adverse effects on employment that have been noted. ${ }^{18}$ It is therefore important to ensure that pain is routinely assessed in such high risk groups. Although tools for assessing pain in this needy population have recently been developed, their clinical utility and sensitivity have yet to be determined. ${ }^{11}{ }^{12}$ There is therefore an urgent need to develop robust assessment measures that can be used in everyday clinical practice to routinely detect pain and monitor the effectiveness of pain relief programmes in these children. Without accurate assessment, appropriate pain relief cannot be provided.

This work was supported by a grant from Sport Aiding medica Research for KidS (SPARKS). 1 Zacharias M, Watts D. Pain relief in children. BMF

2 Goldman A. Pain management. Arch Dis Child 1993;68: 423-5.

3 Perquin CW, Hazebroek-Kampschreur AA, Hunfield JA, et al. Pain in children and adolescents: a common experience. Pain 2000;87:51-8.

4 Perquin CW, Hazebroek-Kampschreur AA, Hunfeld JA, et al. Chronic pain among children and adolescents; physician consultation and medication use. Clin F Pain 2000;16:22935 .

5 Oberlander TF, O'Donnell ME, Montgomery CJ. Pain in children with significant neurological impairment. $\mathcal{F}$ Dev Behav Pediatr 1999;20:235-43.

6 Fanurik D, Koh JL, Schmitz ML, et al. Children with cognitive impairment; parent report of pain and coping. $\mathcal{F}$ Dev Behav Pediatr 1999;20:228-34.

7 Anand KJS, Stevens B, McGrath P, eds. Pain in neonates, nd revised and enlarged edition. Amsterdam: Elsevier, 2000.

8 Lenton S, Stallard P, Lewis M, Mastroyannopoulou K. Prevalence and morbidity associated with non-malignant Prevalence and morbidity associated with non-malignant Dev. 2001;27:389-98.

9 McGrath PJ, Finley GA, Ritchie J. Parents' roles in pain assessment and management. IASP Newsletter March/April 1994:3-4.

10 Manne SL, Jacobsen PB, Redd WH. Assessment of acute paediatric pain: do child self report, parent ratings and nurse rating measure the same phenomenon? Pain 1992;48:45-52.

11 Stallard P, Williams L, Velleman R, et al. Brief report: behaviours identified by caregivers to detect pain in non-communicating children. F Pediatr. In press.

12 Breau LM, Camfield C, McGrath PJ, et al. Measuring pain accurately in children with cognitive impairments: refineaccurately in children with cognitive impairments: r
ment of a caregiver scale. $\mathcal{F}$ Pediatr 2001;138:721-7.

13 Breau LM, McGrath PJ, Camfield C, et al. Preliminary validation of an observational pain checklist for persons with cognitive impairment who are unable to verbally communicate. Dev Med Child Neurol 2000;42:609-16.

14 Fanurik D, Koh JL, Schmitz ML, et al. Pain assessment and treatment in children with cognitive impairment: a survey of nurses' and physicians' beliefs. Clin F Pain 1999;15:30412 .

15 Finley GA, McGrath PJ, Forward SP, et al. Parent's management of children's pain following "minor" surgery. Pain 1996;64:83-7.

16 Malleson PN, Connell H, Bennett SM, Eccleston C. Chronic musculoskeletal and other idiopathic pain syndromes. Arch Dis Child 2001;84:189-92.

17 Hotopf M, Carr S, Mayou R, et al. Why do children have chronic abdominal pain, and what happens to them when they grow up? Population based cohort study. BMF 1998;316:1196-200.

18 Mastroyannopoulou K, Stallard P, Lewis M, Lenton S. The impact of childhood non-malignant life threatening illness on parents: gender differences and predictors of parental adjustment. $\mathcal{F}$ Child Psychol Psychiatry 1997;38:823-9. 